\title{
Interactive comment on "The seasonal cycle of ice-nucleating particles linked to the abundance of biogenic aerosol in boreal forests" by Julia Schneider et al.
}

\section{Anonymous Referee \#1}

Received and published: 28 August 2020

This study shows the seasonal cycle of immersion-mode ice nucleating particles (INPs) in boreal forests in Finland. Since few studies have reported the seasonal cycle of atmospheric INPs in a forested site based on year-round measurements, the INP datasets presented here are unique and will be valuable for atmospheric science communities. This study also suggests that the seasonal cycle of INPs would be attributable to biogenic aerosol particles and provides two new INP parameterizations related to ambient temperature and ice nucleation active site (INAS) approach. However, since evidences to support these points are insufficient, the authors should conduct an indeep discussion based on other available datasets (e.g., meteorological data, WIBS and L-RoF-AMS data) to quantify the possible contributions of biogenic aerosol parti- 
cles to INP populations. I would like to support the publication if the authors can answer the following requests/comments.

Major Comments:

1) Further detailed discussion is necessary to investigate a possible relationship between INPs and primary biological aerosol particles (PBAPs). For example, this study uses ambient temperature data as a proxy of changes of the season. As illustrated in Figure 4, it seems that the variation of INPs active at given temperatures are somewhat related to that of ambient temperatures. On the other hand, the comparison with other meteorological parameters is not performed, despite the fact that many earlier studies (e.g., Heald and Spracklen, GRL 2009; Hoose et al., ERL 2010; Huffman et al., ACP 2013; Wright et al., Aerosol Sci. Technol. 2014) have suggested that humidity would be a key factor influencing the release of PBAPs and/or INPs. In addition, as cited in the Introduction section of this paper, rain events might also be an important factor. The authors should conduct further comparison with other meteorological parameters.

2) In addition to the meteorological parameters and snow coverage/depth, the authors might need to check indicators showing the seasonal cycle of vegetation in/around the monitoring site (if available). For example, some earlier studies have used leaf are index (LAI) data as a measure of vegetation density (e.g., Heald and Spracklen, GRL 2009; Hoose et al., ERL 2010).

3) After checking the points described in the above Comments 1 and 2, I would like to suggest reconsidering the INP parameterization related to ambient temperature (equation 1). I admit that this parameterization seems to successfully reproduce the INP number concentrations at this site as compared with other existing parameterizations. However, it is skeptical if this parameterization is scientifically meaningful, while it may be technically useful for reproducing only INP number concentrations near the ground level of this site. Given that this study emphasizes the possible contribution of PBAPs to INP populations, the development of parameterizations related to PBAPs and/or 
factors influencing the release of PBAPs would be more scientifically valuable. For example, given that previous model studies have used humidity and LAI to simulate the release of PBAPs, it might be interesting to evaluate if these parameters would also be useful for developing new INP parameterizations.

4) As for the datasets during the HyICE-2018 campaign, the WIBS data would be potentially powerful, because these data might be useful as an indicator of biogenic aerosol particles. Unfortunately, however, the explanations and discussion on these data are insufficient. The authors should add detailed explanations and discussion regarding the WIBS data. For example, the WIBS has multiple channels as illustrated in Figure A2, but I could not find any detailed explanations and discussion about these data in the main text. I assume the categorization, such as FL1, FL2, and FL3, is based on Savage et al. (AMT, 2017). If so, the authors should clarify the source of this definition and then clearly explain the characteristics of each channel provided in Figure A2 (for example, what materials are expected to be detected using FL1, FL2, FL3, and their combinations?). In addition, the authors may need to discuss what channel was most sensitive to the variations of INPS.

5) The explanations on the L-RoF-AMS data are also insufficient. The authors should briefly explain what kinds of organics were measured using the L-RoF-AMS and how they obtained organic mass concentrations presented in Figure 3.

Specific Comments:

6) I could not find any descriptions of the objective of this study in the Introduction section. I thought that most sentences in the first paragraph of the Methods section (Lines 75-88) should be included in the Introduction section.

7) Sections 2.1: What is the top height of the inlet? It is also important to provide the information on vegetation of the SMEARII site (e.g., tree canopy heights, dominant vegetation types) and the difference of the heights between the inlet and canopy. 
8) Line 189 (and Figure 3): What is the definition of snow coverage? Where and how was the snow coverage measured?

9) Line 190: The authors would need to briefly explain about how pollen and other PBAPs reported by Manninen et al. (2014) have been measured at the SMEARII site.

10) Line 208 (and Figure 4b): What is the definition of snow depth? Where and how was the snow depth measured?

11) Figure 1: The size of numbers in $x$-axis and $y$-axis are too small.

12) Figure 3a: What is the unit of $y$-axis? As for pollen and PBAP data, since pollen is a kind of PBAPs, I think these should be treated as "pollen" and "other PBAPs". Also, since the pollen and other PBAP data were obtained in 2003/2004, these data should not be included in Figure 3a. I would like to suggest making another figure if they want to show the pollen and other PBAP data in 2003/2004.

13) Figure $3 b$ : What is the unit of $y$-axis? What is the source of fluorescent particles in this figure? I guessed that it would be the same as "all" in Figure A2 according to a description "all three lase-channel combinations of WIBS" in the main text (lines 205206). However, these curves in Figures $3 b$ and Figure A2 seems to be quite different. Please clarify this point (see also Comment 4).

14) Figure 3b: I would like to suggest including the data on the number and/or surface area concentrations in PM10 (the data in Figure A1) during the HylCE period in this figure. Then, the authors should discuss if the variations of INPs are indeed related to organics and fluorescent particles, rather than PM10 particles.

15) Figure 6d: How did the authors apply the parameterization by Ullrich et al. (2017)? Did they measure the surface area concentrations of mineral dust particles at this site?

Or did they simply apply this parameterization to the measured total aerosol surface area concentrations in PM10? Please clarify this point.

Discussion paper

16) Figure A1a: I could not understand how to see this figure. What do the authors 
mean by "percentage deviation"? Please clearly explain the meaning of this word.

17) What are the valid temperature ranges of equations 1 and 2? Did you apply these equations to all available INP data or INP data in a specific temperature range when you made Figure 7?

Interactive comment on Atmos. Chem. Phys. Discuss., https://doi.org/10.5194/acp-2020-683, ACPD 2020. 